\title{
GPPS-BJ-2019-0207
}

\section{LOSS ASSESSMENT OF THE AXIAL-GAP SIZE EFFECT IN A LOW-PRESSURE TURBINE}

\author{
Marcel Oettinger, Dajan Mimic, Michael Henke \\ Institute of Turbomachinery and Fluid Dynamics \\ Leibniz Universität Hannover \\ oettinger@tfd.uni-hannover.de \\ Hanover, 30167, Germany
}

\author{
Oleg Schmunk \\ MTU Aero Engines AG \\ oleg.schmunk@mtu.de \\ Munich, 80995, Germany
}

\author{
Joerg R. Seume \\ Institute of Turbomachinery and Fluid Dynamics \\ Leibniz Universität Hannover \\ Hanover, 30167, Germany
}

\begin{abstract}
The aim of this work is the decomposition, quantification, and analysis of losses related to the axial gap size effect. Both experimental data and unsteady RANS calculations are investigated for axial gaps equal to $20 \%, 50 \%$ and $80 \%$ of the stator axial chord. A framework for identifying sources of loss typical in turbomachinery is derived and utilized for the low-pressure turbine presented. The analysis focuses on the dependency of these losses on the axial-gap variation. It is found that two-dimensional profile losses increase for smaller gaps due to higher wake-mixing losses and unsteady wake-blade interaction. Losses in the end-wall regions, however, decrease for smaller gaps. The total system efficiency can be described by a superposition of individual loss contributions, the optimum of which is found for the smallest gap investigated. It is concluded that these loss contributions are characteristic for the medium aspect-ratio airfoils and operating conditions investigated. This establishes a deeper physical understanding for future investigations into the axial-gap size effect and its interdependency with other design parameters.
\end{abstract}

\section{INTRODUCTION}

The axial spacing between moving and stationary rows has been a major focus of research over the past years. This trend is driven by the need to optimize unsteady blade-row interaction and meeting design goals such as more compact components and reduced weight.

The axial gap size affects the intensity of intra-row secondary flow, wake and potential interactions. Pressure fluctua- tions between moving and stationary rows can be subdivided into a uniformly steady component, a steady component relative to the stator's reference frame and a steady component relative to the rotor's reference frame. According to Jung (2000), the component which is steady relative to one frame acts as an unsteady fluctuation relative to the other component.

Praisner et al. (2006) argued that a larger axial gap would be beneficial regarding system loss, as the wakes mix out to a higher degree before they are affected by dilation resulting in higher loss. The wake impinging upon the leading edge can result in increased incidence and can induce bypass transition (Coull and Hodson, 2011); the intensity of both effects is inversely proportional to the axial gap size.

For three-dimensional flow, however, secondary flow caused by the end-wall boundary layers has to be considered as well. As a result of the losses induced by secondary flow vortices, as described, e.g., by Denton (1993), smaller axial gaps appear beneficial in this regard.

At the mid-span, Pichler et al. (2017) were able to identify higher losses for smaller axial gaps using quasi threedimensional LES simulations. Because of decreasing potentialfield-interaction effects and resulting lower wake dilation, the production of turbulent kinetic energy decreases, thus confirming the arguments of Praisner et al. (2006).

For a shrouded rotor with an aspect ratio of $\mathrm{AR}=2.3$, Yamada et al. (2009) found an optimal gap of $37 \%$ stator chord at the design point. The near-hub losses increased with axial gap size and the tip-region losses decreased, because of stronger and weaker passage vortices, respectively. 
In the studies conducted by Restemeier et al. (2013) and Gaetani et al. (2010), secondary flow intensity was the dominant factor causing loss. Both of the turbines investigated feature comparatively small aspect ratios and an unshrouded design, resulting in stronger secondary-flow losses than profile losses. An optimum was found at a gap equal to $50 \%$ of the stator axial chord. Similarly, Park et al. (2003) noticed higher efficiency for a smaller axial gap due to the decreasing intensity of secondary flow.

While the basic aspects of the axial gap size effect - endwall losses tend to increase with gap size, while profile losses typically decrease-are generally well-understood, a strong disagreement regarding the influence of axial gap size on system loss can be identified in the literature. One reason for conflicting results is the general interdependency of the loss contributions as well as the axial gap size effect correlating with secondary design parameters. Bellucci et al. (2017) found a strong interdependency of axial-gap related losses with both aspect ratio and inlet Reynolds number. In a review of the literature, Grönman et al. (2014) were able to correlate the axial-gap size effect with secondary parameters like the stator's pitch-toaxial-chord ratio, circumferential Mach number, and the rotor aspect ratio. Additional dependencies, such as the rotor being shrouded or unshrouded, cavity design or blade loading, are likely to exist as well.

Utilizing both experimental data and numerical results obtained from unsteady RANS calculations, this paper aims to show the development and sources of loss for three different axial gaps. Accounting separately for individual loss contributions in a quantitative manner forms the basis for future investigations into the interdependency of the axial-gap size effect with other design parameters and operating conditions. To this end, the present paper will answer the following three questions:

1. How does the total loss relate to the axial gap?

2. How do individual loss contributions depend on gap size?

3. What are the underlying physical effects?

\section{Loss Analysis}

The most accurate and robust method to quantify loss is the assessment of the total amount of entropy present in a thermodynamic system. Since the system considered is modeled using adiabatic wall boundaries, entropy as per

$$
s=c_{p} \ln \left(\frac{T}{T_{\text {ref }}}\right)-R \ln \left(\frac{p}{p_{\text {ref }}}\right)
$$

lends itself as the base quantifier of flow losses. In order to separate the losses according to their generating flow phenomena, distinct quantities for identification are needed. The distinction between different sources of entropy is based purely on phenomenological criteria and not on physical entropy-generating mechanisms, i.e., the losses are distributed amongst profile boundary layers, wakes, end-wall boundary layers (hub and tip), and secondary flow.

A common feature which all these flow phenomena share, is that they exist in flow regions where the vorticity $\vec{\omega}=\vec{\nabla} \times \vec{c}$ does not equal zero. The first distinction can be made between secondary flow and boundary layers as well as wakes. Secondary flow consists of vortex structures which can be characterized by their streamwise vorticity

$$
\omega_{\mathrm{SW}}=\frac{\vec{\omega} \cdot \vec{c}}{|\vec{c}|},
$$

which is the projection of the vorticity vector onto the local velocity vector. Using the Pythagorean theorem, the vorticity vector can be decomposed into a streamwise and a crosswise component perpendicular to the local flow direction

$$
\vec{\omega} \cdot \vec{\omega}=\omega^{2}=\omega_{\mathrm{SW}}^{2}+\omega_{\mathrm{CW}}^{2}
$$

The crosswise vorticity is characteristic for boundary layers, wakes, and rotational shear layers in general. Based on these considerations, the following blending factors can be formulated:

$$
w_{\mathrm{SW}}=\frac{\omega_{\mathrm{SW}}^{2}}{\max \left(\omega^{2}, 1 \cdot 10^{-16}\right)}
$$

and

$$
w_{\mathrm{CW}}=\frac{\omega_{\mathrm{CW}}^{2}}{\max \left(\omega^{2}, 1 \cdot 10^{-16}\right)} .
$$

The subdivision between profile, end-wall boundary layer and wakes, however, necessitates additional blending factors. To discriminate between end-wall and profile boundary layers or wakes, the vorticity in cylindrical coordinates $\vec{\omega}=\left(\omega_{x}, \omega_{r}, \omega_{\theta}\right)^{T}$ is used and the following differentiation between cases is defined:

$$
\psi_{r}(\vec{\omega})= \begin{cases}1 & \text { if } \omega_{r}^{2}>\left(\omega_{x}^{2}+\omega_{\theta}^{2}\right) \\ 0 & \text { else. }\end{cases}
$$

Here, $\psi_{r}(\vec{\omega})$ represents a Boolean function, which is 'true' or unity, when the local vorticity vector is of predominantly radial orientation, i.e., in the profile boundary layers and wakes. The entropy due to crosswise vorticity of radial orientation is

$$
s_{\mathrm{CW}, r}=w_{\mathrm{CW}} \psi_{r} s
$$

and

$$
s_{\mathrm{CW}, \neg r}=w_{\mathrm{CW}}\left(1-\psi_{r}\right) s
$$

for deviating orientation of the vorticity vector. 
To further isolate the profile boundary layers from the wakes, an distinction is made, based upon the distance normal to the profile $d_{\mathrm{P}}$ :

$$
\psi_{\mathrm{P}}\left(d_{\mathrm{P}}\right)= \begin{cases}1 & \text { if } \quad d_{\mathrm{P}} \leq \delta_{\max } \\ 0 & \text { else. }\end{cases}
$$

The critical distance of $\delta_{\max }=0.6 \mathrm{~mm}$ is chosen, which is the medium boundary layer thickness across all blades of the present configuration.

Regions, where $\psi_{r}(\vec{\omega})$ returns 'false' or zero are consequently characterized by predominantly axial-circumferential vorticity. This is the case in the end-wall boundary layers. But also in some parts of the wakes, as they interact with secondary flow and become in parts twisted amongst the longitudinal axes of the secondary flow vortices. The separation between hub and tip boundary layer and the part due to wakes is defined by the relative radial height $\left(r-r_{\min }\right) / H$ :

$$
\psi_{\mathrm{H}}\left(\frac{r-r_{\min }}{H}\right)= \begin{cases}1 & \text { if } \quad \frac{r-r_{\min }}{H}<0.15 \\ 0 & \text { else }\end{cases}
$$

and

$$
\psi_{\mathrm{T}}\left(\frac{r-r_{\min }}{H}\right)= \begin{cases}1 & \text { if } \frac{r-r_{\min }}{H}>0.90 \\ 0 & \text { else. }\end{cases}
$$

The regions between $\left(r-r_{\min }\right) / H \approx 0.15$ and $\left(r-r_{\min }\right) / H \approx$ 0.90 are included in the wake losses.

The individual contributions to the overall entropy are, therefore, defined as follows:

The contribution of secondary flow is given by

$$
s_{\mathrm{SW}}=w_{\mathrm{SW}} s
$$

the profile boundary layer by

$$
s_{\mathrm{CW}, \mathrm{P}}=w_{\mathrm{CW}} \psi_{\mathrm{P}} \psi_{r} s
$$

the hub boundary layer by

$$
s_{\mathrm{CW}, \mathrm{H}}=w_{\mathrm{CW}}\left(1-\psi_{\mathrm{P}}\right)\left(1-\psi_{r}\right) \psi_{\mathrm{H}} s ;
$$

the tip boundary layer by

$$
s_{\mathrm{CW}, \mathrm{T}}=w_{\mathrm{CW}}\left(1-\psi_{\mathrm{P}}\right)\left(1-\psi_{r}\right) \psi_{\mathrm{T}} s ;
$$

and the contribution of the wakes is determined by

$$
s_{\mathrm{CW}, \mathrm{W}}=w_{\mathrm{CW}}\left(1-\psi_{\mathrm{P}}\right)\left(1-\psi_{\mathrm{H}}\right)\left(1-\psi_{\mathrm{T}}\right) s .
$$

Each individual loss contribution is then integrated over the main flow domain to obtain the resulting total, i.e.,

$$
S_{i}=\iiint_{\mathcal{V}} \rho s_{i} \mathrm{~d} V
$$

The approach shown here is used to assess shock-wave vortex interaction in Mimic et al. (2019) where it is found to produce similarly satisfactory results.

\section{TEST CASE}

The general flow field of the present 1.5-stage turbine configuration was investigated previously by Henke et al. (2016) and Biester et al. (2013). A primary goal of this turbine design was to ensure a homogeneous flow field in the mid-span unaffected by the end-walls. It is therefore suitable for quantifying losses according to the decomposition above.

\section{Turbine Configuration}

The turbine is a low-pressure turbine with an aspect ratio of $\mathrm{AR}=2.15$ as described by Henke et al. (2016). Both stator rows can be moved in axial direction as well as be circumferentially clocked independently from each other (Henke et al., 2012). Three different axial gaps were chosen for experimental investigation. These gaps $X$ are equal to $20 \%, 50 \%$ and $80 \%$ of the stator axial chord, respectively. Both axial spacings are adjusted in unison for both the stator-rotor and rotor-stator gap. The clocking is adjusted together with axial-gap variation by changing the relative circumferential position between the stator blade rows in order to achieve a similar incoming wake distribution relative to the blades and to isolate the axial-gap size effect: an imaginary particle originating from the stator 1 trailing edge impinges upon the stator 2 leading edge for all configurations. As the blade count of stator 2 is double that of stator 1 (Tab 1), only every second blade of stator 2 passage is impinged upon by the stator 1 wake flow.

Detailed aerodynamic characteristics and a base verification of the overall time-averaged flow field can also be found in Henke et al. (2016).

\section{Instrumentation}

The instrumentation of the experimental setup has been devised in order to answer the above questions in terms of time-accurate and time-averaged measurements of the local flow conditions. In order to measure the overall efficiency, rake probes measuring total pressure and total temperature at the inlet and outlet as well as pressure instrumentation on the blade surfaces were designed during a numerical pre-test analysis, including a local optimization of measurement positions.

Table 1 Test conditions and stage characteristics

\begin{tabular}{llll}
\hline Rotational speed $n$ in $1 /$ min & & 6950 & \\
Inlet total pressure $p_{\mathrm{t} \text {,in }}$ in $\mathrm{kPa}$ & & 168.6 & \\
Inlet total temperature $T_{\mathrm{t}, \text { in }}$ in $\mathrm{K}$ & & 344.2 & \\
Mass flow rate $\dot{m}$ in $\mathrm{kg} \mathrm{s}^{-1}$ & & 8.9 & \\
Total pressure ratio $\Pi_{\mathrm{t}}$ & & 1.42 & \\
\hline & Stator 1 & Rotor & Stator 2 \\
Number of blades & 18 & 30 & 36 \\
Aspect ratio AR & 2.15 & 2.15 & 2.15 \\
Reynolds number Re & $7.5 \cdot 10^{5}$ & $6.5 \cdot 10^{5}$ & $4.2 \cdot 10^{5}$ \\
\hline
\end{tabular}


Figure 1 shows a sectional view of the flow path including measurement planes (MP). MP00, which is located further upstream of the control volume and thus not shown here, features equally spaced rake probes to verify the homogeneous, swirl free inlet flow of the turbine on a grid of six circumferential rakes with six radial positions each. Rake probes in MP32 have been designed to deliver a $360 \mathrm{deg}$ record of total quantities as well as the static pressure and swirl angle. In order to operate these rakes in the three-dimensional flow field downstream of stator 2, the Kiel-head-type probe heads have been designed in a pre-twisted manner in order to record accurate data of the local flow-field for all radial measurement positions.

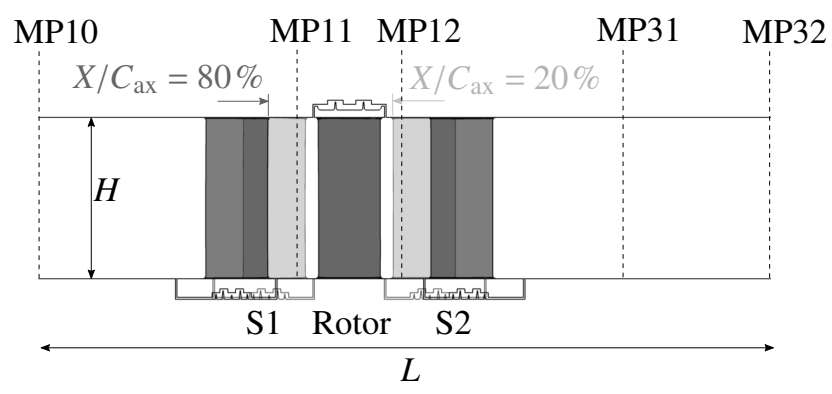

\section{Figure 1 Schematic of the 1.5-stage turbine test rig Small gap $X / C_{\mathrm{ax}}=20 \%$ in light grey; large gap $X / C_{\mathrm{ax}}=80 \%$ in dark grey.}

As these rake probes provide only limited radial resolution with regard to the flow field, additional five-hole-probe measurements have been conducted in measuring planes MP10 (inlet section) and MP31 (outlet section). While rake probes allow continuous data acquisition, probe traverses provide better resolution in radial direction for a specific moment in time. A combination of these data sources provides a set of highresolution boundary conditions for each experiment, which are used in the present work. Within the stator-rotor and rotor-stator gap regions between the blade rows, time-averaged as well as time-resolved multi-hole-probe traverses can be taken for the medium and large axial gap configuration. For the smallest gap investigated, the remaining gap between two blade rows is too small for probe measurements. Therefore, the blading itself has been equipped with pressure taps (time-averaged and time-accurate Kulites) on the suction and pressure sides and in the leading and trailing edges. This allows to determine the wake-flow properties and unsteady flow characteristics for all three axial gap configurations.

\section{Numerical Setup}

The experimental data is supplemented by steady and unsteady RANS calculations for all three gaps using the TRACE solver, which is being developed by the German Aerospace Center (DLR) in cooperation with MTU AeroEngines AG. The general numerical setup used in this work is mostly identical to the ones described in Henke et al. (2016) and Biester et al. (2012). The following short summary will focus on modifications to the referenced setups.

For steady-state calculations, mixing planes are positioned at $50 \%$ of the axial gap. For unsteady simulations, one blade passing period (BPP) is resolved by 1152 time steps per period; each time step is resolved using 30 sub-iterations. A CFLnumber of 50 is used for all calculations. As a result of the blade counts detailed in Tab. 1, a periodic 60 deg-segment is used for the simulations. The Wilcox $1988 k$ - $\omega$-model (Wilcox, 1998) with the extension by Kato and Launder (1993) to correct the turbulent kinetic energy production near the stagnation point is used to model turbulence. The extension of Bardina et al. (1985) is used to account for rotational effects. A multimode transition model as described by Kožulović et al. (2007) is used to model transition. Viscous walls in the main flow domain utilize a low-Reynolds formulation of the turbulence model. Walls inside the cavities are not resolved; instead, wall functions are used locally.

To assess convergence, the fluctuating pressure signal at several point probes inside the computational domain is evaluated. Convergence is reached, when the maximum amplitude deviation between two periods is below a threshold of $\Delta p<0.1 \%$. With the exception of static pressure-which is area-averaged-all flow variables are mass-averaged across the specified planes.

\section{FLOW FIELD ANALYSIS}

In line with the introductory discussion, the flow field is decomposed into a quasi two-dimensional flow at the mid-span and three-dimensional flow across the entire span. In order to obtain a better understanding of the loss evolution, axial distributions are considered as well.

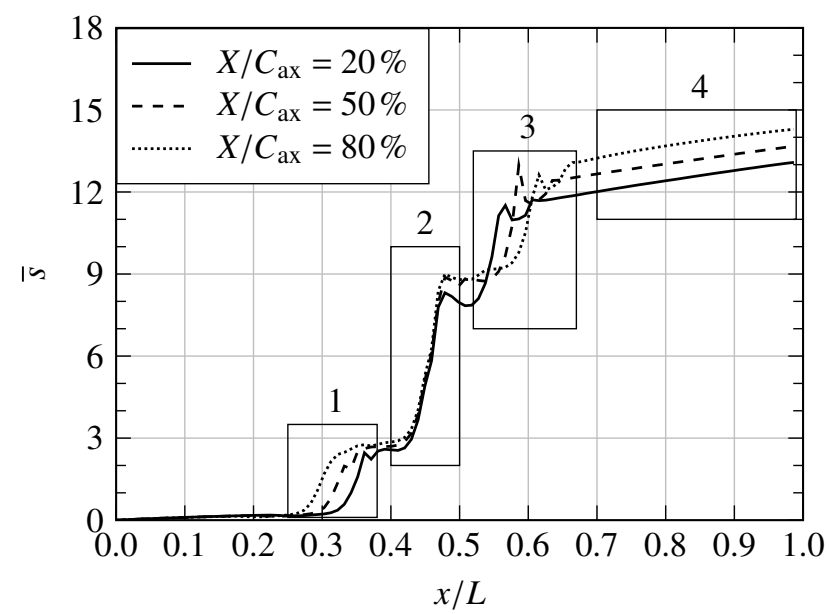

Figure 2 Axial distributions of time-averaged entropy (URANS calculations). 


\section{Axial Distributions}

Figure 2 depicts the axial distribution of circumferentially and spanwise-averaged entropy for all gaps. Four regions of interest are identified:

1. Stator 1: Initially, losses are proportional to the flow path length upstream of the stator 1 leading edge. Downstream of the first stator, losses increase initially for larger axial gaps as a result of increased secondary-flow and wakemixing losses.

2. Rotor: All axial gaps show a nearly identical slope in entropy increase. For the smallest gap, total losses at the rotor trailing edge are substantially lower than for the other gaps.

3. Stator 2: Due to the stronger wake-boundary-layer interaction at the second stator, losses increase for the smaller gaps.

4. Flow downstream of stator 2: Wakes and vortices mix out increasingly, resulting in linearly increasing losses. The slope is flat relative to the absolute difference between axial gaps, i.e., compensating smaller axial gaps with an outlet further upstream - as would be the case for neighboring components in a real machine-does not result in equal total system loss.
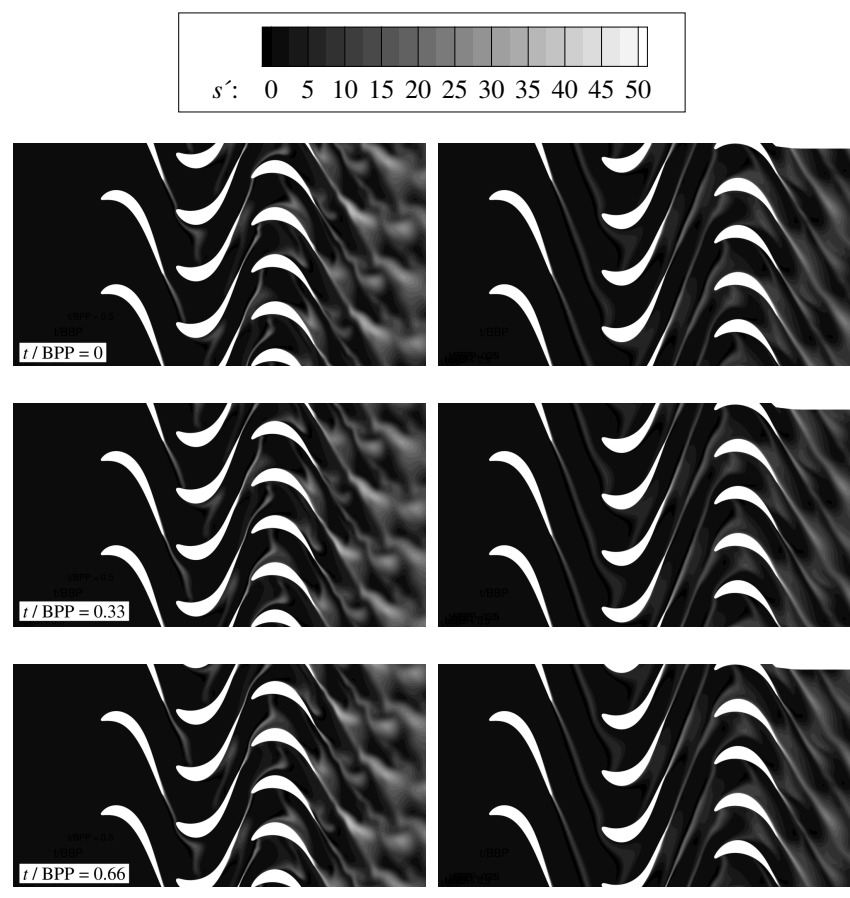

Small gap $X / C_{\mathrm{ax}}=20 \%$

Large gap $X / C_{\mathrm{ax}}=80 \%$

Figure 3 Instantaneous entropy contours at mid-span (URANS).

Since cavities are not included in the post-processing control volume, entropy fluctuations at the cavity inlets and outlets can be observed, which can also result in a local entropy decrease in the main flow domain. Generally speaking, the entropy increase

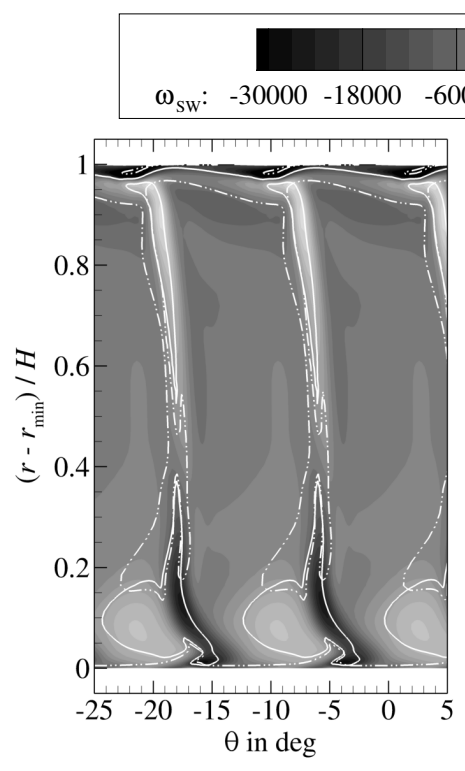

Medium gap $X / C_{\mathrm{ax}}=50 \%$

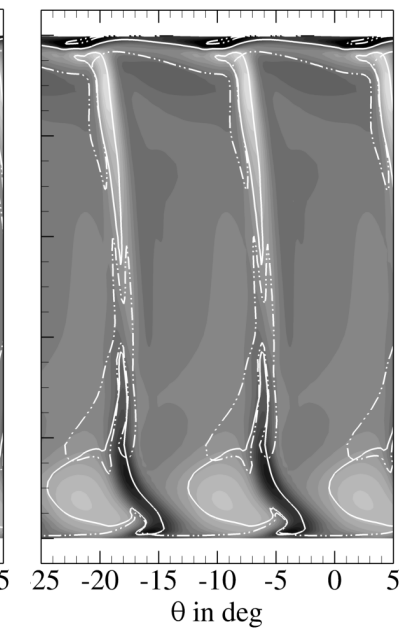

Figure 4 Time-averaged flow field downstream of the rotor (MP12, URANS). Dash-dotted lines denote $s_{\mathrm{CW}}$; solid lines $s_{\mathrm{SW}}$.

is steepest in the rotor and stator passages and less pronounced outside of the blading. Minimum system loss occurs for the smallest axial gap, while losses are highest for the maximum gap examined. The underlying physical effects are investigated in further detail below.

\section{Two-Dimensional Flow Field}

For the quasi two-dimensional flow field at mid-span, profile losses are the dominating factor. Instantaneous entropy contours at different time steps are pictured in Fig. 3 to visualize the wakes for the small and large gaps. The low-momentum wakes of the first stator enter the rotor, experience dilation and are transported into the second stator frame. Because of the comparatively high turning, an increase in axial gap affects the wake mixing process disproportionately. For the large axial gap, the wake is almost entirely mixed out when entering the rotor, the same is true for the rotor wakes entering the second stator. For the small gap, wakes are deflected by the leading edge of the downstream row almost as soon as they are shed; a strong interaction of the wake with the potential field of the downstream row can be inferred. Downstream of the second stator, a highly unsteady flow field can be identified, which is caused by the comparatively strong wake inducing periodic fluctuations on the suction side; wakes of varying intensity are shed. As a result, entropy production at mid-span increases considerably for the smallest axial gap. This evaluation is consistent with the two-dimensional prediction of Praisner et al. (2006).

\section{Three-Dimensional Flow Field}

The basic aspects of the three-dimensional flow field in the form of circumferentially averaged flow variables were 

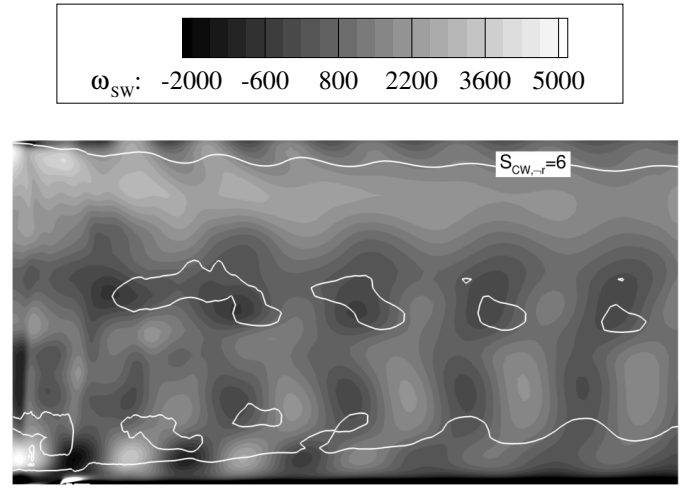

Small gap $X / C_{\mathrm{ax}}=20 \%$

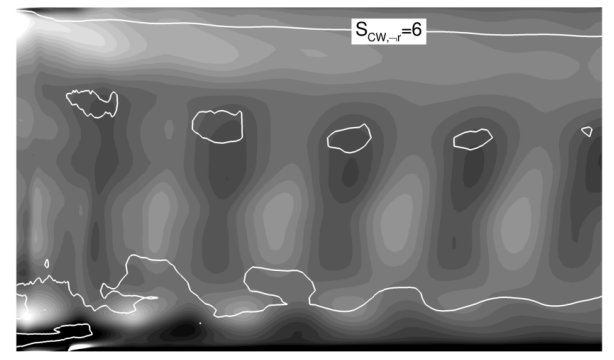

Large gap $X / C_{\mathrm{ax}}=80 \%$

\section{Figure 5 Circumferentially and time-averaged streamwise vorticity downstream of stator 2 featuring loss contribution $s_{\mathrm{CW}, \neg r}$ (URANS).}

presented by Henke et al. (2016). Figure 4 depicts distributions of streamwise vorticity in measurement plane (MP) 12 . With the distance between rotor trailing edge and MP12 remaining constant despite gap variation, both gaps show only minor differences regarding secondary flow, represented here by the streamwise vorticity and the streamwise loss component $s_{\mathrm{SW}}$. The circumferential extent of the hub passage vortex increases slightly for the larger gap, reflecting its dependency on the upstream flow conditions. Regarding wake-related losses in the mid-span region, as represented by the crosswise loss component $s_{\mathrm{CW}}$, a slight increase is found for the medium gap. This is due to smaller axial gaps increasing the wake interaction between the upstream stator and the rotor, as is also evident in Fig. 3. This results in increased loss being transported downstream by the wake. The functional decomposition of entropy using streamwise and crosswise vorticity is capable of identifying individual sources of loss. The hub and tip wall boundary layers are captured using $s_{\mathrm{CW}}$. In the presence of trailing shed vortices, an overlap of both contributions exists in the wake region. An interaction between the vortices and the wakes can be inferred, as the streamwise loss component virtually cuts into the crosswise-dominated region. The presence of $s_{\mathrm{SW}}$ in the tip region is a result of shroud leakage flow re-entering the main flow.

Figure 5 depicts the distribution of circumferentially averaged streamwise vorticity downstream of the second stator. Ad- ditionally, a single contour line of the loss contribution $s_{\mathrm{CW}, \neg r}$ representing the crosswise loss component not aligned in the radial direction, is shown. Naturally, $s_{\mathrm{CW}, \neg r}$ is non-zero at the end walls, allowing for the identification of end-wall boundary layer related losses. Of particular relevance is the shear layer caused by cavity-main-flow interaction at the hub, which merges with the boundary layer and is identified as the main driver for end-wall losses. End-wall-related losses therefore increase with gap size and the hub-related contribution is higher than the tip-related contribution. Despite $s_{\mathrm{CW}, \neg r}$ being, by definition, equal to zero if the local vorticity vector is oriented in radial direction - as is the case for ideal, two-dimensional profile boundary layers and wakes-a non-zero contribution of this fraction can be identified in the mid-span region. Addi-

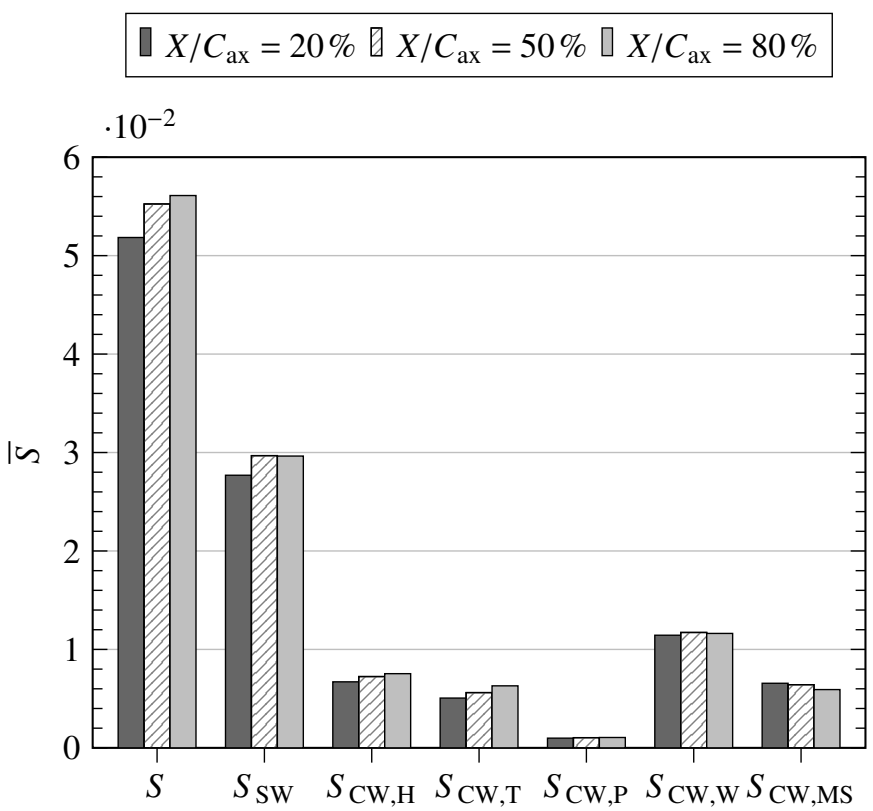

\section{Figure 6 Loss contributions from time-averaged URANS.}

tional spots of increased $s_{\mathrm{CW}, \neg r}$ are visible in the lower third just above the hub wall boundary layer. Here, wake interaction with the vortices results in vortex deflection, where the vortices are virtually cut by the passing wake. This also causes torsion of the wake, which is the reason behind the non-zero contribution of $s_{\mathrm{CW}, \neg r}$ outside of the boundary layers. This effect is more pronounced for the smallest axial gap. As can be seen in Fig. 3, the flow area influenced by the wake is much larger for the small gap as a result of the highly unsteady wake shedding at the second stator, which exceedingly amplifies wake torsion and resulting vortex deflection. The spots of increased $s_{\mathrm{CW}, \neg r}$ near the hub merge with the hub boundary layer into one continuous flow region. This impedes a clear distinction between both unique effects. The blanking merely based on radial distance to the end walls reveals potential for improvement of the loss decomposition proposed. 


\section{INTEGRAL PARAMETERS}

\section{Loss Analysis}

Based on the detailed flow analysis, losses are broken down in Fig. 6. Total system loss increases with axial gap size for the present study. These results are consistent with Yamada et al. (2009), who investigated a similar turbine configuration. In the hub and tip regions, more pronounced boundary and shear layers from cavity interaction increase losses. As is evident in Fig. 5, end-wall losses at the hub are higher than at the tip which is also reflected in Fig. 6. Profile losses, i.e., the losses immediately caused by the blade boundary layers, are a negligible loss factor and remain almost constant regardless of axial gap. Interestingly, wake losses remain almost constant for all axial gaps. As can be seen in the efficiency distribution in Fig. 7, a greater flow-path length - as is the case for a decrease in axialgap size-results in increasing redistribution of total enthalpy from the mid-span into the endwall regions. This effect is superposed by the aforementioned merging of end-wall boundary layer and wake structures. Since wake-torsion losses are only counted between $15 \%$ and $90 \%$ relative channel height, as per the definition above, a redistribution into the boundary layers results in an underestimation of wake-induced losses, i.e., wake losses are counted as end-wall losses in the loss decomposition proposed. This is due to end-wall and wake-torsion losses being both represented by $s_{\mathrm{CW}, \neg r}$. A closer look at the wake losses at the mid-span between $40 \%$ and $70 \%$ relative channel height, which are labeled $S_{\mathrm{CW}, \mathrm{MS}}$ in Fig. 6, reveals an increase in wake-induced losses inversely proportional to the axial gap; this is consistent with the observed increase in $s_{\mathrm{CW}, \neg r}$ in the mid-span region (Fig. 5) and the efficiency distribution in Fig. 7. The exact cut-off between the end-wall and mid-span regions as well as the distinction between inter-merged effects can be improved for a better loss prediction.

The losses observed in the mid-span region can be subdivided into direct wake-blade interaction, mixing losses and wake torsion with resulting vortex deflection downstream of the blades. As is shown in Fig. 3, the maximum increase in entropy actually occurs downstream of stator 2 and not inside of the blade passages, leading to the conclusion that the latter two effects are dominant with regard to wake losses.

The nearly identical secondary flow losses for the medium and large axial gap suggest that the small axial gap disproportionally inhibits the formation of secondary flow vortex structures. If the gap reaches a certain threshold, secondary flow losses remain mostly constant, the effect of the increased flow path length is reflected in the end-wall boundary layer losses. Considering Fig. 5 and the decomposition above, it can be argued that the increase in secondary flow losses occurs primarily at the tip region downstream of the second stator. For the investigated aspect ratio and operating conditions, end-wall related losses (secondary flow and endwall boundary layers) account for roughly $2 / 3$ of the total losses.

\section{System Efficiency}

Figure 7 depicts the flow field at the outlet of the control volume for all axial gaps investigated. Of particular note is the missing total pressure measurement at $93 \%$ relative channel height for the large gap. As the total pressure information is missing for 2 circumferential positions, no representative average can be calculated. Because of this, and a similar problem for the total temperature measurement at $9 \%$ relative channel height, no efficiency information at these positions can be given for the large gap case. For the isentropic efficiency, a clear distinction of the axial-gap size effect depending on radial location can be identified in accordance with the loss breakdown presented: In the hub and tip regions, losses increase for larger gaps. In the tip region in particular, a clear distinction can be made, which is consistent with the loss breakdown: While the hub shows higher loss in general, the variation at the tip is higher than at the hub. In the region between $40 \%$ and $70 \%$ relative channel height an inverse trend can be identified, i.e., losses increase for smaller gaps. Of particular note is the aforementioned redistribution from the mid-span region towards the end-wall regions. The small-gap distributions are, therefore, more uniform compared to the larger gaps. The trends discussed apply to both numerical and experimental data although different predictive trends exist at some radial positions. While the good agreement in the total pressure distribution validates the loss breakdown above, the comparatively high measurement uncertainty, however, precludes a detailed assessment of the experimental efficiency distribution.

$$
\begin{aligned}
& \diamond \text { Exp. } X / C_{\mathrm{ax}}=20 \%-\text { URANS } X / C_{\mathrm{ax}}=20 \% \\
& \triangle \text { Exp. } X / C_{\mathrm{ax}}=50 \%-\text { URANS } X / C_{\mathrm{ax}}=50 \% \\
& \square \text { Exp. } X / C_{\mathrm{ax}}=80 \% \cdots \cdots \cdots \text { URANS } X / C_{\mathrm{ax}}=80 \%
\end{aligned}
$$
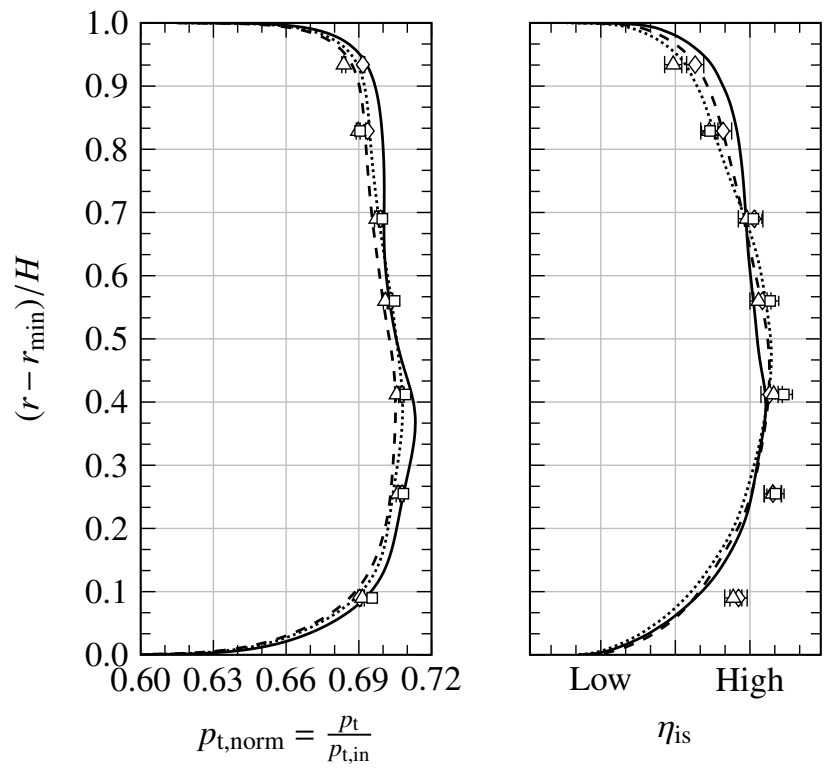

Figure 7 Circumferentially and time averaged outlet flow field for investigated axial gaps. 
The resulting system efficiency is depicted in Fig. 8. In addition to the URANS simulations, results from steady-state calculations are also considered. Despite steady-state CFD not capturing stator-rotor interaction, the overall trend does agree: The optimum gap is found for the smallest gap investigated.

\section{- Time-averaged URANS $\Delta$ Steady RANS}

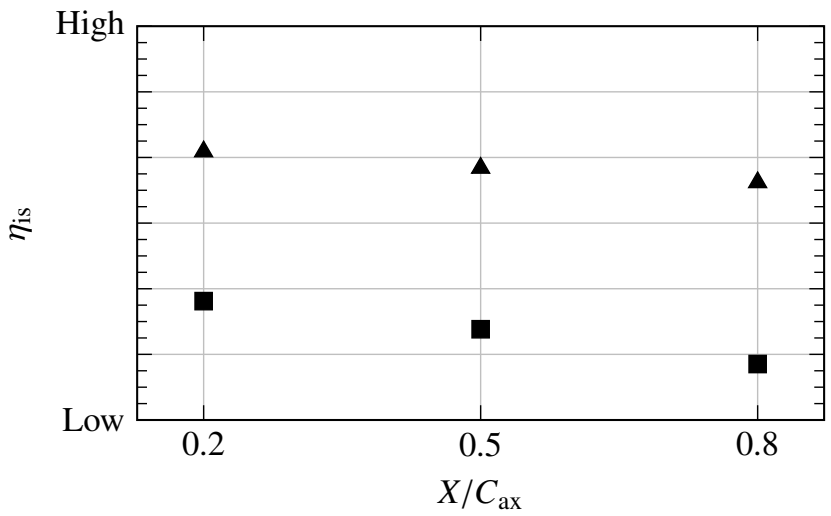

Figure 8 Total-to-total system efficiency.

\section{CONCLUSIONS}

The effect of the axial-gap size on system losses is quantified using a functional separation of different entropy sources, such as end-wall friction, profile loss, and secondary flow. For these individual terms, different trends with regard to axial gap variation can be identified. For the quasi two-dimensional flow field in the mid-span region, larger axial gaps are beneficial due to reduced wake dilation and associated mixing losses in the following row. For the smallest gap, unsteady wake-blade interaction is a major source of two-dimensional loss. A more extensive investigation of the flow region outside of the boundary layers revealed, however, that a clear distinction into twoand three-dimensional flow is valid only for a very small flow region at mid-span. All other flow region are afflicted by a strong interaction between - or even merging of - various phenomena, which are not just a superposition of multiple effects. In contrast to the trend immediately at mid-span, end-wall regions show an increase in losses for larger axial gaps, due to higher friction and stronger secondary flow. End-wall related losses contribute almost $2 / 3$ of the entire system loss: it is found to be the dominating loss contribution.

The optimal axial gap is, therefore, an ideal compromise between individual loss contributions for a given turbine configuration. For the configurations investigated, the optimal gap equals $20 \%$ of the stator axial chord length.

Based upon the framework derived in the present work, the interdependency of the axial gap size effect and secondary design parameters will be the subject of further research. It is, for example, inherently evident that a higher aspect ratio would lead to profile losses becoming a more dominant factor, which would then shift the optimal gap towards a larger size.

Nevertheless, a more refined distinction criterion between endwall boundary-layer and wake flow should be derived before proceeding further.

Since the focus of this paper was the time-averaged flow field, instantaneous unsteady effects were generally neglected. Unsteady losses, therefore, remain a factor to be considered in greater detail.

\section{ACKNOWLEDGMENTS}

We gratefully acknowledge the substantial contribution of the DLR Institute of Propulsion Technology and MTU Aero Engines AG for providing TRACE.

\section{NOMENCLATURE}

\section{Latin symbols}

AR aspect ratio

d distance

C chord

$H \quad$ channel height

$L \quad$ total length of configuration

$\dot{m} \quad$ mass flow rate

$n \quad$ rotational speed

$p \quad$ pressure

$r \quad$ radial coordinate

$R \quad$ gas constant

$S \quad$ specific entropy

$S \quad$ entropy

$t$ time

$T \quad$ temperature

$x \quad$ axial coordinate

$X \quad$ axial spacing

\section{Greek symbols}

$\begin{array}{ll}\delta_{\max } & \text { critical distance } \\ \zeta & \text { loss fraction } \\ \eta & \text { efficiency } \\ \theta & \text { circumferential coordinate } \\ \mu & \text { viscosity } \\ \Pi & \text { pressure ratio } \\ \omega & \text { vorticity }\end{array}$

\begin{tabular}{ll}
\multicolumn{2}{l}{ Subscripts } \\
ax & axial \\
$\mathrm{CW}$ & crosswise \\
$\mathrm{H}$ & hub \\
in & quantity at inlet \\
is & isentropic \\
norm & normalized quantity \\
$\mathrm{P}$ & profile \\
$\mathrm{SW}$ & streamwise \\
$\mathrm{T}$ & tip \\
$\mathrm{t}$ & total quantity \\
$\mathrm{W}$ & wake
\end{tabular}




\section{Operators}

[] time-averaged quantity

[ ] instantaneous quantity

$[\overrightarrow{]} \quad$ vector

\section{Abbreviations}

BPF blade passing frequency

BPP blade passing period

CFL Courant-Friedrichs-Levy number

MP measurement plane

$\mathrm{S} \quad$ stator

Re Reynolds number

\section{References}

[1] Jung, A. "Berechnung der Stator-Rotor-Wechselwirkung in Turbomaschinen". PhD Thesis. Stuttgart: Universität Stuttgart, 2000.

[2] Praisner, T. J., Clark, J. P., Nash, T. C., Rice, M. J., and Grover, E. A. "Performance Impacts Due to Wake Mixing in Axial-Flow Turbomachinery". In: Proceedings of the ASME Turbo Expo 2006. ASME. 2006, 1821-1830. DOI: $10.1115 / \mathrm{GT} 2006-90666$.

[3] Coull, J. D. and Hodson, H. P. "Unsteady boundary-layer transition in low-pressure turbines". In: Journal of Fluid Mechanics 681 (2011), 370-410. Dor: 10.1017/ j fm . 2011.204.

[4] Denton, J. D. "Loss Mechanisms in Turbomachines". In: Journal of Turbomachinery 115 (1993). The 1993 IGTI Scholar Lecture, pp. 621-656.

[5] Pichler, R., Michelassi, V., Sandberg, R., and Ong, J. "Highly Resolved LES Study of Gap Size Effect on LowPressure Turbine Stage". In: Journal of Turbomachinery 140.2 (2017). Dor: 10.1115/1.4038178.

[6] Yamada, K., Funazaki, K., Kikuchi, M, and Sato, H. "Influences of Axial Gap Between Blade Rows on Secondary Flows and Aerodynamic Performance in a Turbine Stage". In: Proceedings of the ASME Turbo Expo 7 (Jan. 2009), pp. 1039-1049. DoI: 10.1115/GT200959855.

[7] Restemeier, M., Jeschke, P., Guendogdu, Y., and Gier, J. "Numerical and Experimental Analysis of the Effect of Variable Blade Row Spacing in a Subsonic Axial Turbine". In: Journal of Turbomachinery 135.2 (Mar. 2013). DOI: $10.1115 / 1.4006587$.

[8] Gaetani, P., Persico, G., and Osnaghi, C. "Effects of Axial Gap on the Vane-Rotor Interaction in a Low Aspect Ratio Turbine Stage". In: Journal of Propulsion and Power 26.2 (2010), 325-334. Dor: 10. 2514/1 . 37616.

[9] Park, J., Choi, M., and Baek, J. "Effects of Axial Gap on Unsteady Secondary Flow in One-Stage Axial Turbine". In: Journal of Turbo and Jet Engines 20 (2003), 315333.

[10] Bellucci, J., Rubechini, F., Arnone, A., Arcangeli, L., Maceli, N., Paradiso, B., and Gatti, G. "Numerical and Experimental Investigation of Axial Gap Variation in
High-Pressure Steam Turbine Stages". In: Journal of Engineering for Gas Turbines and Power 139.5 (2017). DoI: $10.1115 / 1.4035158$.

[11] Grönman, A., Turunen-Saaresti, T., Röttä, P., and Jaatinen-Värri, A. "Influence of the axial turbine design parameters on the stator-rotor axial clearance losses". In: Proceedings of the Institution of Mechanical Engineers, Part A: Journal of Power and Energy 228.5 (2014), pp. 482-490. DoI: 10.1177/0957650914531949.

[12] Mimic, D., Sauer, P., Oettinger, M., and Herbst, F. "Shock-Vortex Interaction in a Transonic Turbine Cascade". In: Proceedings of Global Power and Propulsion Society Beijing Conference 2019. GPPS-BJ-2019-0008. 2019.

[13] Henke, M., Wein, L., Kluge, T., Guendogdu, Y., Biester, M. H., and Seume, J. R. "Experimental and Numerical Verification of the Core-Flow in a New Low-Pressure Turbine". In: Proceedings of the ASME Turbo Expo 2016. ASME. 2016, DoI: 10.1115/GT2016-57101.

[14] Biester, M. H.-O., Wiegmann, F., and Guendogdu Y.and Seume, J. "Time-Resolved Numerical Study of Axial Gap Effects on Labyrinth-Seal Leakage and Secondary Flow in a LP Turbine". In: Proceedings of the ASME Turbo Expo 2013. ASME. 2013. DoI: 10. 1115/GT201395628.

[15] Henke, M., Biester, M. H.-O., Guendogdu, Y., Lippl, F., Mass, E., and Seume, J. R. "Numerical assisted Design of a Variable Rotating Vane Carrier Device for Turbine Test Rigs with split Housing Structures using a Segmented Half-Ring Bearing Concept". In: Proceedings of the 53rd AIAA/ASME/ASCE/AHS/ASC Structures, Structural Dynamics and Materials Conference. AIAA. 2012. DOI: $10.2514 / 6.2012-1660$.

[16] Biester, M. H., Henke, M., Seume, J. R., Guendogdu, Y., and Engel, K. "Unsteady Wake-Blade Interaction: A Correlation Between Surface Pressure Fluctuations and Loss Generation". In: Proceedings of the ASME Turbo Expo 2012. Vol. 8. ASME. 2012, 2743-2752. DoI: 10. $1115 /$ GT2012-69616.

[17] Wilcox, D. C. Turbulence Modeling for CFD. 2nd ed. DCW Industries, 1998.

[18] Kato, M. and Launder, B. E. "The modeling of turbulent flow around stationary and vibrating square cylinders". In: 9th Symposium on Turbulent Shear Flows. 1993, pp. 10.4.1-10.4.6.

[19] Bardina, J., Ferziger, J. H., and Rogallo, R. S. "Effect of rotation on isotropic turbulence: computation and modelling". In: Journal of Fluid Mechanics 154 (1985), 321-336. DOI: 10.1017/SOQ22112085001550.

[20] Kožulović, D., Röber, T., and Nürnberger, D. "Application of a Multimode Transition Model to Turbomachinery Flows". In: Proceedings of the 7th European Conference on Turbomachinery. 2007, 5-9. 